\title{
Researching Chinese-English Geological Translation Strategies in China Based on the Translational Writing Framework
}

\author{
Yanhong $\operatorname{Pan}^{1 *}$
}

\author{
${ }^{1}$ Minnan Science and Technology Institute, Nan'an, Fujian, China \\ *Corresponding author. Email: panyanhong@mku.edu.cn
}

\begin{abstract}
This research paper is a study in China of the Chinese-English translation of geological articles, especially the reports of geological exploration projects, by exploring their theoretical basis. It also aims to suggest the adoption of the translational writing mode for better rendering of such articles. The author collected throughout the CNKI website and categorized relevant literature published in China's core journals from 1954 to 2016, and summarized the content and theoretical application of such previous research. In addition, after studying Nord's notion "function plus loyalty" and Yang's "perception - through operation - expression — review" mode of translational writing, the author believes that they are good guides to both practice and academic research of Chinese-English geological translation. Translators should enhance their writing ability, with a strong awareness of being a writer to reach high translation quality.
\end{abstract}

Keywords: Geological Report, Chinese-English Geological Translation Strategies, Translational Writing Framework, Function plus Loyalty

\section{INTRODUCTION}

The translation covers many fields and permeates various disciplines, including geology, literature, dramas, etc. Zheng points out that China began with geology in the dissemination of evolutionary ideas [1]. The importance of geology to the humanities is evident, and translation, among other things, has contributed to the development of geology. Against the background of globalization and increasing international cooperation in various industries, Chinese mining enterprises also follow the development trend of the times and go global for international cooperation. The geological exploration industry's trend to go global is bound to boost the demand for Chinese to English (for short, C-E) geological translation. This paper attempts to explore the translation of geological articles, especially the reports of geological exploration projects, based on the research of their theoretical basis and to suggest the adoption of the mode of translational writing for better rendering of such articles.

\section{THEORETICAL FRAMEWORK}

\subsection{Research on China's Geological Translation Studies and Its Findings}

The geological industry in China has been developing at the turn of the 21 st century. The demand for geological translation is an important part of the translation market, which cannot be underestimated. According to a survey conducted by Miao et al. on the translation industry trend in terms of fields, geology ranks 12th among the 20 professional fields in demand in the translation market [2].

The author collected throughout CNKI and categorized relevant literature published in China's core journals from 1954 to 2016 (as of June 2016). With the subject term "translation" accompanied by "geology," and the discipline "philosophy and humanities," 64 articles were retrieved, though 33 articles were not related to translation knowledge and skills. Thus, after screening, there were 31 journal articles on geological translation study. 
Table 1. Statistics of Research Content

\begin{tabular}{lll}
\hline Content & $\begin{array}{l}\text { Number } \\
\text { of } \\
\text { articles }\end{array}$ & Percentage \\
\hline $\begin{array}{l}\text { Translation of geological } \\
\text { terminology }\end{array}$ & 4 & $13 \%$ \\
$\begin{array}{l}\text { Tips/strategies of geological } \\
\text { translation }\end{array}$ & 9 & $29 \%$ \\
$\begin{array}{l}\text { Translation of geological } \\
\text { landscape (especially }\end{array}$ & 8 & $26 \%$ \\
$\begin{array}{l}\text { slogans) } \\
\begin{array}{l}\text { EST (English of science and } \\
\text { technology) teaching for }\end{array}\end{array}$ & 2 & $6 \%$ \\
$\begin{array}{l}\text { geology } \\
\text { Translation of geological } \\
\text { papers }\end{array}$ & 4 & $13 \%$ \\
$\begin{array}{l}\text { Others } \\
\text { Total }\end{array}$ & 4 & $13 \%$ \\
\hline
\end{tabular}

The data show few studies on the translation of geological texts in China. Such texts are generally classified under the broad category of science and technology translation, rather than a separate topic for translational discussion. Although "geological translation is an important medium for information exchange between Chinese geological scientists and their foreign counterparts, its research has long been at the edge of translation" [3], without an established research system. Among the few geological translation studies, the most popular text for translation is geological landscape information, accounting for $26 \%$, followed by professional geological papers or their abstracts and titles (13\%); nine articles (29\%) are summaries about geological translation techniques/strategies alone, generally based on practical experiences of geological insiders or those who have been engaged in geological translation for a long time; four articles specifically discuss the translation of specialized geological terminology.

It can be seen that the research on geological C-E translation in China mainly focuses on specialized/nearspecialized vocabulary, nominalization, passive sentences, long and difficult sentences, and that it is limited within academic geological literature and tourist landscape introduction, without further research on geological texts of other genres, such as reports of geological exploration projects which are commonly seen during the actual international communication of the geological community.

Table 2. Summary of the Theoretical Basis of Each Relevant Study

\begin{tabular}{ll}
\hline Theoretical basis & $\begin{array}{l}\text { Number } \\
\text { of } \\
\text { articles }\end{array}$ \\
\hline $\begin{array}{l}\text { Functionalist theory } \\
\text { Faithfulness, expressiveness, and } \\
\text { elegance (or faithfulness to the original) }\end{array}$ & 4 \\
$\begin{array}{l}\text { Equivalence } \\
\text { Semantic translation and communicative } \\
\text { translation }\end{array}$ & 2 \\
\hline
\end{tabular}

\begin{tabular}{ll}
\hline The principles of accuracy, clarity, & 2 \\
conciseness, and standardization & 2 \\
Cultural transfer & 1 \\
Information equivalence and transferability & 1 \\
Lexicography & 3 \\
The unity of theory and practice & 1 \\
Journal editorial requirements for & \\
standardization & 9 \\
No clear articulation of theories used & \\
\hline
\end{tabular}

It is found that among the journal articles that focus on geological translation strategies and techniques, some do not clearly point out their guiding theories of translation but only combine the characteristics of special geological English and specifically summarize and analyze the use of various techniques. As these 9 translation articles focus more on practice, they might unconsciously utilize several translation theories but not indicate it out consciously. This will not affect their translation as they have already conveyed the meaning of the original text. The functionalist theory is more commonly used in geological translation due to the emphasis on translation purposes, faithfulness, and fluency. Their translation focuses on how to express meaning by language to achieve specific functions.

He et al. propose that C-E translation for geology should be based on: 1) a basic understanding of both the knowledge of earth science and the structure of the original text, 2) accurate wording, and also 3 ) flexible use of translation skills in the unity of theory and practice, with lasting efforts in learning and improvement [4]. These are general insights emphasizing the unity of theory and practice for professional translation. However, Peng highlights the functionalist theory, pointing out that the theory bases itself on the intended purpose and function of the target text, considers the differences in text types as well as the needs and cognitive ability of the readers of the translated text, breaks through the limitations of the original language text, and has feasibility in translating tourism materials of geological landscape [5]. Similarly, Yuan et al. conducted statistical analysis on the abstracts of papers published in authoritative foreign geological journals and found that the English abstracts of British and American people feature simple sentences, more active than passive voice, and basically present tenses, with fewer past tenses and no future tenses. They propose the purpose-based translation strategy for abstracts of geological papers based on statistical analysis results [6].

In addition, to understand the use of functionalist theory in the translation of geological texts, the author searched for related literature published in core journals throughout CNKI. By using "translation" and "geology" as the search terms and "Skopos" as the full-text search condition, 12 results were obtained, 6 journals, and 6 master's theses. When "functionalism" was used as the search term, 7 results were obtained, all of which were included in the above 12 results. Combined with the data in the above table, it can be seen that the functionalist 
translation theory is often used as a theoretical guide to geological C-E translation practice.

In summary, the author's main findings are as follows: (1) research on geological C-E translation in China is still limited, and there is no special research on texts produced in actual geological exploration (e.g., survey reports); (2) the theory commonly used in geological translation research is the functionalist theory, which mostly emphasizes translation purposes, faithfulness, and fluency, and fails to further look at the act of translation from the perspective of the translator's relationship with the principal or client beyond the textual sphere, and at the interpersonal interaction in translation practice, which reflects loyalty to their principals or clients.

\subsection{Literature Review}

\subsubsection{Introduction of Nord's Notion "Function Plus Loyalty"}

In the 1990s, Christina Nord, in Translating as a Purposeful Activity - Functionalist Approaches Explained, was the first to comprehensively and systematically summarize the ideas of the functionalist in English, clarifying the academic theories and terminology of the functionalists, and putting forward the principle of loyalty, which improved the German functionalist theory [7]. In his book Text Analysis in Translation, Nord first proposed "function plus loyalty." "Function" refers to the factors that make the target text function in the desired way in the target text; "loyalty" refers to the interpersonal relationship between the translator, the original-text sender, the target-text recipient, and the initiator [7]. According to Nord, "loyalty" is a responsibility of the translator to his or her partners in the translation interaction and must be distinguished from "fidelity/faithfulness" because "fidelity/faithfulness" usually refers to the relationship between the original text and the target text. In contrast "loyalty" belongs to the interpersonal sphere and refers to "social relations between people" [7]. The translator needs to negotiate with other parties involved in the translation task, making sure to be loyal to both the reader and the initiator of translation and the text producer, reconciling the original culture and the target culture.

Nord's notion "function plus loyalty" focuses on translator training. Nord identifies three aspects of the functionalist theory that are particularly useful for translation training, namely, "the role of the translation brief, the role of original-text analysis, and the classification of translation problems" [7]. Specifically, the translation brief contains "a) the (intended) text function(s), b) the target-text addressee(s), c) the (prospective) time and place of text reception, d) the medium over which the text will be transmitted, and e) the motive for the production or reception of the text", all of which are considered before the actual translation [7].
The original text analysis guides the translation process by helping the translator to determine (1) whether the translation task is feasible, (2) which units of information in the original text are functionally relevant to the translation, and (3) what translation strategies will enable the translation to meet the requirements of the translation brief [7]. In contrast, translation problems should be addressed in top-down order, i.e., the functionalist translation process should start at the pragmatic level, that is, the intended function of the translation should be determined first; then the type of translation should be considered to ensure that the translation style conforms to the cultural conventions of the source or translated language; then differences in language systems, contexts, and even lower-level personal preferences of the translator should be considered, but the function of the translation should always be respected in any case [7].

All in all, Nord's notion "function plus fidelity" requires the translator to pay attention to the elements of the translation brief, to the analysis of the original text, and to the priority over dealing with translation problems. All this is to achieve a translation practice that is more faithful to the parties involved in the translation task, while always respecting and being faithful to translation functions.

\subsubsection{Introduction to Yang's Mode of Translational Writing}

The concept of "translational writing study" is mentioned for the first time in the "Preface" of Yang Shizhuo's English-Chinese Translation Course (Peking University Press, 2006), Chapter 8 of which is devoted to the possibility of constructing "translational writing study." In 2008, Yang published the Towards a Theory of Translational Writing in Issue 3 of Writing, explaining the idea of constructing translational writing study. In his 2012 monograph English-Chinese Translational Writing Study, Yang systematically discusses his translational writing study, pointing out that the decisive factor for the merit of a translation is the translator's translational writing ability. Drawing on and applying the basic principles of writing science- "perception-thought operation-expression," as well as the Western writing process model, Yang proposes the translation process of "perception - thought operation-expression-review" [8]. Combining the Western translation theory and the traditional Chinese translation theory, the construction of the translational writing study aims to pursue the translation process of "translating at will without exceeding the rules," respect translation principles and standards, and on this basis, give full play to the linguistic advantages of the target language and show the translator's translational writing ability [9]. One of its research tasks is to expound the laws of translational writing, guide the practice of translational writing, avoid 
blindness and enhance self-awareness [9]. The process of translation is as follows:

Perception: refers to selecting the original material and the understanding of the original text [9]. First, the translator must select a suitable translation text and initially perceive the original text to determine whether he or she is competent for the translation task. If blindly, it may harm the client's interests. Second, the translator must understand the original text, perceive the original work's genre and structure, the writing style of the original text, the content of the original text, and the target language's cultural environment [9].

Thought operation: refers to using the target language to think of the information in the original text. In this process, the translator should first determine the author's intention, secondly grasp the original text's information and style (including style), and find the tone of the original text. Besides, he or she should also find out the clues of the original text and follow its organizational structure [9].

Expression: both expressing in the target language and making the translation perfect. The translator must follow (and coordinate) the person and perspective of the original, pay attention to the logic, syllables, and tone of the original expression, control personal preferences, and express according to the original message, style, and target audience. The translation should be revised and checked several times afterward to ensure that the original text's message is faithfully conveyed, and that the language is naturally fluent, even beautiful [9].

Review: refers to re-examine the translation, correct it according to certain criteria to make it perfect, and evaluate whether the translation properly conveys the content, form, and style of the original, and correct any inappropriate parts [9].

The above four stages are interconnected; the translator has thought operation while perceiving the original text; constant perception and thought operation enable him/her further to express, and review is even done throughout the translation process.

The translational writing study has been affirmed and highly evaluated by translation research scholars such as Guo Jianzhong, Feng Qinghua, and $\mathrm{Wu}$ Jianping. Although it currently focuses on English-Chinese translation, it guides translators on how to "translate well" and "translate subtly," which is of great significance to translation practice for improved translation quality. Guo has also proposed five levels of translation concepts and methods on translation language operation: free translation of words, phrase substitution, sentence reconstruction, paragraph restructuring, and chapter rewriting. "Whether it is a free translation, substitution, reconstruction or reorganization, rewriting is needed, and its theoretical basis is that there are huge differences in grammar and lexical structures between English and
Chinese, and its emphasis and argument is also the importance of the translator's ability to write in the target language" [10]. Liu also proposes that "reconstruction" ('rewriting') is the most radical alternative to solve the obstacles of word order and expression layers, and is also the best substitute to ensure the readability of the target language, and is obviously the most convenient to take advantage of the translation" [11]. It can be seen that there are not few discussions on the ability to write in the target language and the advantage of the target language, so Yang's translational writing study is of great significance to both translation's academic research and practice.

\subsubsection{Summary}

In this chapter, the author presents her survey on geological translation research in China and summarizes such previous research's content and theoretical application. Also, after introducing Nord's notion "function plus loyalty" and Yang's "perception-thought operation-expression-review" model of translational writing, the author holds that they are a good guide to both practice and academic research of Chinese-English geological translation.

\section{RESULTS AND DISCUSSIONS}

\subsection{Characteristics of English Translation of Chinese Reports on Geological Exploration Projects}

This section is going to summarize the findings of translation characteristics based on the collected literature that we have observed:

(1) Scientific (technical) report style: The geological exploration report belongs to the scientific (technical) style, with clear expressions, concise and accurate wordings, clear organizations, and ethical and legal regulations. As a report, its form, content, and style should meet the recipient's background and interests of the report.

(2) The translation procedure: The original Chinese text is often long, with even a single sentence becoming a paragraph, truly professional and informative. The translator needs to fully perceive the meaning of the original text, think of it carefully and logically, separate or integrate the original sentences, reorganize and express them with the style of the original text in mind and concisely and accurately that should also conform to the writing characteristics of the report style.

(3) The professional requirements for translators: The original Chinese text may contain some seemingly common but specialized words, such as "prospecting," "overburden," "morphology", and "northwest-southwest (NE-SW)". For a translator who does not have much 
experience in translation and does not know much about the relevant knowledge, he/she should check it with an open mind because many of them are knowledge points and have their specific meanings and translations, which cannot be taken for granted. For those that cannot be determined, the translator needs to take the initiative to consult professionals, clients, customers, etc.

\subsection{The Translation Strategies for Geological Reports and Other Professional Texts Based on the Mode of Translational Writing}

The following section is the author's reflections about the translation of geological reports based on the mode of translational writing and the author's practical experience. Before translation, the translator must understand the writing characteristics and internal logical relationship of a specific genre and give full consideration to it when translating to present a related satisfactory result. During the translation process, the translator had better determine the original author's intentions, consider the style of the original text when thinking about how to convey the message of the original text, and also grasp the organization of the original text to have a clear understanding of the writing idea of the original text. It is also important to follow the logic of the original text, control the translator's preferences, and base his/her presentation on the original message, style, and recipients. Throughout the translation process, the translator should constantly reflect on whether his/her understanding is accurate; whether the vocabulary is correctly chosen; whether the language expression conforms to the norms and customs of the target language; whether the format is correct, etc., and carry out quality control consciously. When the translator is working on professional texts (e.g., geological reports), he/she should be clear about the client and the recipients' requirements, adopt an appropriate language style, and convey the information in the original text accurately and objectively, and ensure clear and strict logic.

\section{CONCLUSION}

To better promote global cooperation and provide better translation services, translators pay attention to professional explorations based on translation theories in practice. They should enhance their writing ability with a strong awareness of being a writer to translate from the perspective of writing, guided by the translational writing mode of "perception - thought operation - expressionreview." However, this is only one of the useful translation strategies that exist in the world. There should be other helpful translation strategies. All these are to make the translation authentic and accurate and better meet the expectations of recipients.

However, the problem is that in translation practice, the translator may undertake the translation of a document alone rather than cooperate with other translators, which makes it possible that individual differences in understanding, writing, and translation leave translation quality unassured. Also, in terms of quality, the concept of "authentic and accurate translation" in translational writing is not an objective standard that is quantified. Due to the above limitations, further research is needed in the future to investigate the standard of "authentic and accurate translation" in translational writing by quantification.

\section{REFERENCES}

[1] Z. Zheng. 1996. One Hundred Books that Have Influenced Modern China. Beijing: China Foreign Translation \& Publishing Corporation.

[2] J. Miao, S. Wang. 2010. Implications of the Employment Trend of Translation Industry for the Education of Master of Translation (MTI). Foreign Languages and Their Teaching, (3): 63-67.

[3] F. Gu. 2012. Stylistic Features and Translation Strategies of Geological English. Chinese Science \& Technology Translators Journal, Vol. 25, (2): 12-14, 36.

[4] D. He, C. He. 2007. On English-Chinese Translation of Geological Documents. Journal of Chengdu University of Technology (Social Sciences), Vol. 15, (4): 96-99.

[5] Y. Peng. 2008. English Translation of Geological Landscape Tourism Interpretation from the View of the Functionalist Theory. Journal of Taiyuan Normal University (Social Science Edition), Vol. 7, 06: 124125.

[6] Y. Yuan, S. Yang. 2013. Translation of Abstracts of Geological Papers. Overseas English, (21): 199-200.

[7] N. Christiane. 2001. Translating as a Purposeful Activity: Functionalist Approaches Explained. Shanghai: Shanghai Foreign Language Education Press. Language Education Press.

[8] J. Zhou. 1989. Advanced Course in Writing. Wuhan: Wuhan University Press.

[9] S. Yang. 2012. English-Chinese Translation Writing. Beijing: China Foreign Translation \& Publishing Corporation.

[10] J. Guo. 2012. Brief Comment on Translational Writing Study. Shanghai Journal of Translators, (3): 79-80.

[11] M. Liu. 2012. Contemporary Studies of Translation. Beijing: China Foreign Translation \& Publishing Corporation. 